wie auch Christus aus den beiden Völkern, aus Freien und Sklaven Kinder Gottes machte. Alles das tat er aber wegen der jüngeren, Rahel, die schöne Augen hatte, und in der die Kirche vorweg angedeutet war. Un ihretwillen hielt Christus große Marter aus, der damals durch die Patriarchen, seine Propheten, das Vorbereitete zuvor andeuten und verkünden ließ.e Sollte nun wirklich Irenäus, - der die Parallelen zwischen Jakob und Christus in solcher Menge aufzuzählen weiß, in diesem einen Stück sich aus dem Dialog bereichert haben, der ihm sonst kaum etwas geboten hätte außer diesem Hinweis auf Rahel als den Typus der Kirche? Einen zwingenden Grund, Irenäus zu dem Abhängigen zu machen, wird man schwerlich ausfindig machen können. Andererseits weist auch nichts auf eine $\mathrm{Ab}$ hängigkeit des Dialogs von Irenäus. Die gemeinsame Quelle für diese allegorischen Erklärungen sind in der schulmäßigen Methode zu suchen, nicht in der Erfindungskraft einer einzelnen Persönlichkeit. Stand die Methode fest, so mußte man mit ihrer Hilfe in der Hauptsache wohl auch zu ähnlichen Deutungen kommen, da sich eben die Allagorese doch immer in ziemlich eng umgrenżten Bahnen bewegte.

[Hiermit endet die Hanaschrift. Den Schluß niederzuschreiben ist dem Verfasser nicht beschieden gewesen.]

\title{
Die Bedeutung des Paulus für den modernen Christen.
}

\author{
Von + Johannes WeiB.
}

Das Thema dieses Aufsatzes ist mir von der Redaktion formuliert worden. Ich würde vielleicht nicht den Mut gehabt haben, mir selber die Aufgabe zu stellen, die darin liegt. Denn allzusehr bin ich von der Schwierigkeit überzeugt, auf diese Frage eine befriedigende Antwort zu geben.

Was heißt in diesem Falle >Bedeutung \&? Soll damit gefragt werden, ob Paulus dem religiösen Leben des Einzelnen in unsern Tagen der Predigt der Kirche, der Schriftlektüre des Frommen noch etwas zu sagen habe? Nun, die Mehrzahl der wahrhaft Gläubigen,

tauscht, wie aus dem Armenier hervorgeht. Nach propter quam sustinuit sind die Worte omnes cruciatus ansgefallen, und der Schluß ist $z u$ lesen: qui tunc quidem per patriarchas suos prophetas praefiguravit et praenuntiavit praeparata praeexercens suam partem

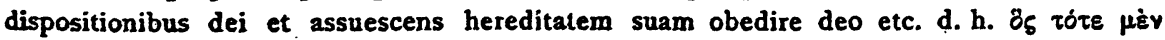

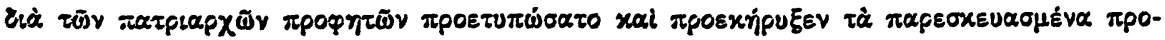

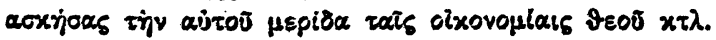


denen die Schrift Licht und Lebensnahrung bedeutet, werden schon die Fragestellung entrüstet zurückweisen, sie werden sich verbitten, wissenschaftlich darüber belehrt zu werden, ob und wie weit Paulus ihrer Seele Führer, Mahner und Tröster sein könne oder dürfe. Das suchende, nach Hilfe und Erbauung verlangende Herz ist unberechenbar; es findet Ansprache da, wo für ein historisches Urteil keine Brücke mehr vorhanden zu sein scheint, geht an Perlen der Religion vorüber, legt sich zurecht, paßt sich an, hört Untertöne und Obertöne heraus, an die kein Exeget jemals gedacht hat, kurz für die wirkliche Frömmigkeit besteht das Problem nicht. Und doch ist sie in Wahrheit die allerschärfste Kritikerin. Mit genialer Urteilskraft scheidet sie das aus, was sie nicht einschmelzen kann, ohne es zu merken und ohne eine Ahnung von dem Radikalismus und Subjektivismus ihres Verfahrens. Es wäre eine großartige Aufgabe, auf dem Wege einer vorsichtigen Enquête einmal festzustellen, wie weit Paulus in unsern Gemeinden wirklich lebt.

So lange wir dies Mittel einer religiösen Experimental-Psychologie nicht haben, bleibt uns nur ein andres Verfahren übrig. Es gilt, die Frage ein wenig anders zu formulieren. Es sind dabei sehr verschiedene Formen denkbar.

Eine in letzter Zeit häufig gestellte Frage lautet: Wie verhält sich Paulus zu Jesus? Ist er wirklich, wie viele annehmen, ein ganz neuer Typus von Religion, der mit Jesus garnichts zu"tun hat, nur ganz äußerlich mit ihm zusammenhängt ? ${ }^{l}$ Oder ist er, bei alle Verschiedenheit der F or m seines religiösen Denkens, doch in Wahrbeit der Fortsetzer der Religion Jesu, mit seinem Empfinden im innersten verwandt, von seinem Seelenfeuer entründet, șein letzter Interpret - wenn auch in einer ganz andern Sprache ${ }^{2}$ Bei dieser Fragestellung wird die Religion Jesu oder wenigstens sein religiöses Grundempfinden als d as Christentum, als die Normalform christlichen Lebens betrachtet, das überall vorhanden sein muß und kann, wo überhaupt der Name , Christ \& noch geführt und ernst genommen wird $^{3}$. Es würde sich sehr verlohnen, diese Frage noch einmal wieder zu untersuchen, und unsere Erörterung wird öfters zu ihr hinüberschweifen. Aber das mir gestellte Thema sieht von Jesus ganz ab und stellt die Frage für Paulus an sich.

Auch bei dieser Beschränkung läßt sie sich verschieden fassen. Man kann fragen:

1 So etwa Wrede, Paulus (Religionsgeschichtl. Volksbücher), Tübingen 1905.

2 So etwa J. Weiß, Paulus und Jesus, Berlin 1909.

3 Dies etwa der Grundgedanke von Harnacks ,Wesen des Christentumss. 
Gibt es.in der Verkündigung des Paulus und in seiner Religion Gedanken, Motive, Empfindungsweisen, die ganz unmittelbar ohne alle historische Vermittlung und alle theologische Reflexion, ohne Umdeutung oder Transponierung in die Denkweise und das religiöse Leben des modernen Christen organisch eingefügt und dort triebkräftig werden können, so daß sie nicht mit seinem sonstigen Weltempfinden oder Denken in Konflikt geraten?

Oder: Ist Paulus nur dadurch für das moderne Christentum lebendig zu machen, daß man die Gemeinden zu einem historisch-psychologischen Verständnis seiner Vorstellungswelt und seines religiösen Lebens erzieht und sie anleitet, an gewissen Punkten durch ein Subtraktionsverfahren oder durch eine Umsetzung in andere Ton.. art oder Gefühlstemperatur, durch Umdenkung in moderne Vorstellungsweise das Antike, Fremdgewordene, Überlebte in ewig Lebendiges, von Jedermann zu Fühlendes zu verwandeln?

Ein geschichtlich denkender Mensch wird sofort sagen: der letztere Weg ist der einzige, auf dem wir nicht nur dem Paulus, sondern jeder Größe der Vergangenheit innerlich nahe kommen können. Es gibt überhaupt keine historische Größse - und stände sie unšzeitlich, national, sprachlich viel näher als Paulus - deren inneres Leben wir ohne weiteres unmittelbar mit dem unsern verbinden können. Schon Luther, wie verwandt wir uns ihm fühlen, wie sehr wir von seiner religiösen Sprache leben, ist uns im Kerne seines Wesens und in seinem mittelalterlichen Denken ungeheuer fern - wie viel mehr Paulus, bei dem zu der antiken Denkweise noch das Trennende einer dunkeln und schwierigen Sprache kommt! Ohne historisches Verständnis und ohne dem entsprechende relativierende, einschränkende Urteile, ohne eine Übersetzung und Ersetzung wird es da nicht abgehen, wenigstens wenn wir auf das Ganze sehen und nach prinzipieller Klarheit streben.

Ich nenne nur einige Punkte, an denen die große Verschiedenheit seines und unsres Denkens besonders deutlich wird:

I. Der ganze Aufriß seines Weltbildes und seiner Erlösungslehre erscheint uns $\mathrm{mythologisch}$. Sein Monotheismus ist in einen dualistis c ben Rahmen gespannt. Der "Gott dieser Welt \&, der Satan, die gottfeindlichen Herrschaften und Gewalten, die persönlichen $>$ Welt-Elemente , die ihm gegenüberstehen, sind für Paulus nichts weniger als abstrakte Schemen, es sind gewaltige Realitäten,

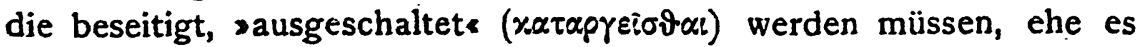
zu der wirklichen Gottesherrschaft kommen kann, da Gott salles in alleme ist. Und das Erlösungswerk ist ein gewaltiger dramatischer

Zeitschr. f. d. neutest. Wiss. 19. Jahrg. $1919 / 20$. 
Kampf zwischen Gott und diesen überirdischen Gewalten: nachdem sie im Kreuzestod über Christus zu triumphieren schienen, hat Gott in der Auferweckung Christi sie im Triumphe aufgeführt, und der erhöhte Christus soll, wie ein Diktator Gottes, sie vollends Gott sunterwerfen«. Die Erlösung des Christen ist keineswegs nur Sündenvergebung oder Gotteskindschaft, sondern Loslösung von den Mächten des Kosmos, durch welche die vorchristliche Menschheit in Sklaverei gehalten wurde; dadurch daß Christus aus himmlischen Sphären in den Bereich der kosmisch-sarkischen Knechtschaft hinabstieg und in seinem Todeskampfe selber die Erlösung von ihnen erfuhr, hat er für die Seinen das Band zwischen Welt, Fleisch und Sünde durchschnitten. - In diesem Gedankengang ist uns Heutigen so gut wie alles fremd geworden; wie ein Stück »Gnosis mutet uns das an. Und wenn wir noch ein Verhältnis dazu haben, können wir es nur, indem wir die bunte, dramatische Fülle jenes Bildes auf den einen abstrakten Gedanken zusammenziehen, daß auch wir glauben, daß Gottes Wille schließlich die letzte triumphierende Macht in der Welt ist und bleiben wird.

2. Die ganze Lehre des Paulus ist $\mathrm{M}$ is si ons- und Bekehrungs Theologie, entworfen von einem Manne, dessen Leben durch einen ungeheuern Umschwung in zwei ganz entgegengesetzte Hälften zerteilt ist: jenseits nur Unseligkeit und Sünde, Knechtschaft, Irrtum, diesseits Freude, Friede, Seligkeit, Kraft, Reinheit, Wahrheit. Alles ist bezogen auf den einen Augenblick, der für alles Spätere grundlegend ist: er hat die Erlösung als ein scharf abgeschnittenes Ereignis erlebt. Und seine Gemeinden, auf die er seine Bekenntnisse und Belehrungen einstellt, bestehen nur aus Menschen, die von einer andern Religion und aus einer gänzlich andern Lebensführung zum Christentum übergetreten sind, die gleichfalls einen Schnitt in ihrem Leben erfahren haben; dies drückt sich schon sprachlich darin aus, daß die religiösen Hauptbegriffe in Form von Verbalsubstantiven auftreten oder als Verba im Präteritum: Erlös ung, Vergebung, Rechtfertig ung, gerechtfert igt, bef $r$ e it, ber ufe $n$, geheil ig $t$

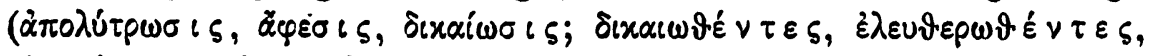
$x \lambda \eta \vartheta \dot{\varepsilon} \vee \tau \varepsilon \varsigma, \hat{\eta} \gamma(\alpha \sigma \alpha \mu \varepsilon \dot{\varepsilon} \vee \iota)$.

$\mathrm{Zu}$ diesen einschneidenden Erlebnissen fehlt es bei der Mehrzahl heutiger Christen, die in der Verkündigung des Evangeliums und christlicher Zucht aufwachsen, an jeder Analogie; es sind doch Ausnahmen, wenn jemand nach einem wie heidnischen Leben sbekehrt* wird; die meisten von uns haben eine Rechtfertigung als einmaligen Akt nie erlebt - wir leben im beständigen Bewußtsein der gött- 
lichen Gnade, die.Sündenvergebung erbittèn und empfangen wir täglich, die Berufung ist uns in die Wiege gelegt und die Heiligung erscheint uns weit mehr als eine dauernde Aufgabe der Selbsterziehung, denn als ein einmaliges Gnadengeschenk Gottes. Am deutlichsten kann man sich das klar machen am Begriff der Gotteskindschaft: bei Paulus erscheint sie als scharfmarkierter Akt der Adoption von Menschen, die bisher keine Gotteskinder waren; wir empfinden unsre Gotteskindschaft - im Sinne Jesu - wie den uns von jeher umgebenden Sonnenschein, dem wir Leben und Glück verdanken, der immer da ist und der immer da war, so daß unsre Aufgabe nur ist, von ihm Gebrauch zu machen.

So können wir uns die Hauptbegriffe des paulinischen Denkens nür durch eine Umdeutung aneignen.

3. Der größte Unterschied besteht vielleicht hinsichtlich der Anschauung vom göt $\mathrm{t}$ lich en Geis te. Für Paulus ist die Verleihung des Geistes ein Erlebnis von aufdringlicher Wahrnehmbarkeit; scharf unterscheidet sich der Pneumatiker vom Nicht-Pneumatiker; der mitgeteilte Geist aber ist etwas ganz Objektives, das sich zunächst in keiner Weise mit dem Innenleben des Christen verschmilzt: „Der Geist s steht unserm > Geiste * wie ein Fremdes gegenüber (Röm 8, 16), sein Zeugnis, sein Abba-Rufen (Röm 8, I5; Gal 4, 5. 6) ist ein Vorgang, den der Christ gewissermaßen als Zuhörer beobachtet; er selbst hat keinen Einfluß darauf. Gerade auf dieser völligen Objektivität beruht die $\mathrm{B}$ ew e is $\mathrm{kr}$ a ft jenes Zeugnisses, daß der Christ sich als Gottes Kind betrachten darf: die Liebe Gottes ist als ein fühlbarer, beseligender Besitz ausgeschüttet in unsern Herzen durch den Geist, der uns gegeben ist, d. h. da d u r ch d a B der Geist uns gegeben ist, besitzen wir ein Unterpfand der göttlichen Liebe in unsern Herzen. Die Form der Vorstellung ist hier ganz antik animistisch - primitiv: es ist ein zweites Geistwesen, das in dem Christen haust. Eine andre Linie wieder verbindet die paulinische Geisteslehre mit hellenistischer philosophischer Mystik. Da erscheint der Geist Gottes als eine aus dem Wesen Gottes in das Innerste des

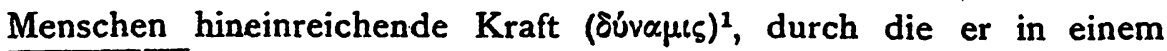

1 Man kann sich das Wesen dieser Vorstellung von göttlichen ovvá $\mu \varepsilon \iota \varsigma$ gut veranschaulichen durch gewisse ägyptische Darstellungen, auf denen der König opfernd erscheint und die Strahlen des Sonnengottes, an ihren Ausläufern Hände tragend, bis an den König heranreichen, einer von ihnen das Lebenszeichen dem Könige unter die Nase haltend (vgl. 2. B. Gressmann, Texte und Bilder S. $66=$ Erman S. 65 ff.). Hier trifft eine ganz primitive Anschauung (der Gott gi bt dem Könige Leben) mit einer erheblich abstrakteren (der König ist durch die vom Gotte ausstrahlenden ouvđáfє! 5 ganz unmittelbar mit ihm sverbunden - wie wir mit einem modernen Bilde aus der Technik sagen mochten) zusammen. 
höhern Sinne ssubstantiell mit Gott und Christus direkt verbunden ist: Christus selbst verschmilzt sich mit ihm zur Einheit mystischen Lebens. Wenn in jener primitiven Gedankenreihe der Geist wie eine Art persönlicher Dämon vorgestellt wurde, so ist hier umgekehrt die persönliche Gottes- und Christus-Vorstellung im Begriffe, sich pantheistisch $\mathrm{zu}$ der. Vorstellung eines göttlichen Fluidums oder der stoischen Weltseele $\mathrm{zu}$ verflüchtigenen ${ }^{1}$. In beiden Fällen aber liegt eine Anschauung zugrunde, die wir uns nicht mehr zu eigen machen können. Wenn wir davon reden, daß wir den Geist Gottes empfangen, so denken wir dabei an eine Stärkung, Reinigung, Erhebung unsrer eigenen Seelenkräfte, die wir durch Gebet oder durch die feste Richtung unsres Glaubenswillens auf Gott gewinnen: Wir denken immanentpsychologisch, und deuten damit sowohl die primitive wie die mystische Gedankenreihe um.

Dies sind nur einzelne Beispiele dafür, daß wir auch die religiösen Grundgedanken des Apostels nur vermöge einer Transposition uns aneignen können. Meist geschieht das unbewußt; der Theologe aber muß wissen, was er tut; er muß in dieser Beziehung prinzipiell klar sein. Wie in diesen Einzelfällen, so wird das Ganze der paulinischen Religion nur in sehr veränderter Form in dem modernen Christen weiterleben können - falls überhaupt ein religiöser Gehalt vorhanden ist, der über die Jahrhunderte weg und trotz der so ganz abweichenden Denkformen auch heute noch Menschen zu fördern und $\mathrm{zu}$ beseligen imstande ist. $\mathrm{D}$ as ist die eigentliche Frage, um die es sich handelt. Wir wagen es, sie zu bejahen und wenigstens an einigen Hauptpunkten, dieses Ja zu begründen: an dem Gottesbegriff des Paulus, an seiner Lehre vom religiösen Grundverhältnis zu Gott, aì seiner Christusfrömmigkeit und an seiner Ethik.

I. Der Gottesgla ube des Paulus hat — wenn man ihn theologisch betrachtet - verschiedene Wurzeln und daher verschiedene Elemente. Um nur die Hauptsachen zu nennen: neben der alttestamentlich-prophetischen Vorstellungsreihe stehen die neuen Züge, die er seiner christlichen Erfahrung verdankt. Man vergesse aber auch nicht den nicht geringen hellenistischen Einschlag, den Paulus aus der Berührung mit dem stoischen Gottesbegriff ${ }^{2}$ mitgebracht hat.

1 Vgl. meine Schrift, Christuse. Die Anfänge des Dogmas. Tübingen 1909. (Religionsgeschichtliche Volksbücher I, 18. 19.)

2 Insbesondere kommt hier wohl Posidonios in Betracht; die pseudo-aristotelische Schrift svon der Welte. 
a) Dieses letztere Moment erscheint mir für die Gegenwart sehr wichtig, wie es ja auch in der Geschichte der Theologie eine ungeheure Rolle gespielt hat. Die Offenbarung Gottes in den Werken der Schöpfung (Röm I, I9 f.), wie Paulus sie als einen freilich nicht genügend beachteten Urbesitz der Menschheit darstellt, und wie sie im Aufriß der christlichen Dogmatik durch Jahrhunderte hindurch als unteres Stockwerk der Religion betrachtet worden ist - dies ist ein Gedanke, der in unserm naturwissenschaftlichen Zeitalter eine neue, noch lange nicht genügend durchdachte Rolle zu spielen berufen ist. Mag man von modern theologischem Standpunkte aus, etwa von der Lehre Ritschls aus; diese Form religiöser Anschauung als etwas Minderwertiges oder als eine Illusion betrachten - es läßt sich nicht leugnen, daß für unendlich viele Menschen hier e in Weg zu Gott ist, den wir ausbauen sollten. Wie Paulus in der Missionspredigt auf ihm die Anknüpfung mit dem gebildeten Heidentum gesucht hat, so ist dies auch für das moderne Christentam eine Brücke zur Verständigung mit der naturwissenschaftlich denkenden Gegenwart. Von einem höhern Standpunkt aus ist es kein Schade, wenn dieser Gedanke keicht eine pantheistische Form annimmt, wie es in der Areopagrede der Apostelgeschichte (17, 23-28) offensichtlich der Fall ist. Wem es um die Erweckung von Religion zu tun ist, darf nicht so engherzig sein, daß er die Anerkennung eines persönlichen Gottesbegriffes zur Bedingung mache. Der Pantheismus mag seine Grenzen und Mängel haben, zweifellos liegt er unsrer nicht nur naturwissenschaftlich, sondern auch künstlerisch angeregten Zeit sehr nahe, und warum soll man nicht diese Form religiösen Lebens, falls es nur lebendig gefühlt und betätigt wird, neben andern Formen anerkennen. Vom Standpunkte des Paulus wenigstens kann man nicht allzuviel dagegen einwenden; denn er kommt (in seiner ChristusPneuma-Lehre) pantheistischen Vorstellungen nahe genug. Aber freilich sein Gottesbegriff enthält noch mehr.

b) Auch da, wo er der hellenistischen religiösen Aufklärung weit entgegenkommt (Röm I, 18-2, 10), hält er doch mit höchster Energie den ethischen Charakter dieses Weltgottes aufrecht. Mit der Offenbarung Gottes in der Natur ist ihm eng verbunden die Offenbarung Gottes im Sittengesetze, das in den Herzen geschrieben steht (Röm 2, 14 f.); derselbe vo0 s, mit dem man den Schöpfergott in seinen Werken erkennt (Röm I, 19), ist auch das Organ für das $>$ Gesetz Gottes (Röm 7, 22 f.). Auch hier mag man vorm Standpunkte sittlicher Erkenntnis aus sagen, daß es kein angeborenes sittliches Bewußtsein gibt, und daß das , Gewissen * eine geschichtlich gewordene 
Größe ist - tatsächlich liegt doch noch - trotz aller Verwilderung - in unsrer Menschheit ein Fond von sittlicher Erkenntnis, ein Rest des Bewußtseins, daß die Welt und der Mensch auf das Gute hin angelegt ist, und wir sind nicht so reich an sittlich-religiösen Werten, daß wir diesen Ansatzpunkt für die Entwicklung höhern Lebens vernachlässigen dürften. Trotz allem, 'was die Dogmatik darüber sagen möge, ist es doch als ein Glück zu betrachten, daß durch Paulus diese Gedanken der Stoa in die christliche Kirche hineingerettet sind und in aller Stille fortwirken, vielfach stärker, als die eigentlich christlichen Gedanken.

c) Vielleicht darf man auch den Vorsehungsglauben des Paulus wenigstens zum Teil als ein hellenistisches Erbstück betrachten. Zwar das stoische Wort Vorsehung « ( $\pi$ póvoc $\alpha$ ) findet sich bei ihm nicht. Aber ein Ausspruch wie Röm 8, 28, den er mit seinem bekannten oíbapev als einen ohne weiteres feststehenden Satz d. h. wie ein Zitat behandelt,

>denen, die Gott li e be n, müssen alle Dinge zum Besten dienen klingt eher hellenistisch als alttestamentlich, jedenfalls nicht eigentlich paulinisch. Es heißt ja nicht ^denen, die Gott liebt « - dies wäre paulinisch gedacht - sondern sdenen die Gott lieben\&, und es ist bezeichnend, daß Paulus, um den Satz seinem Denken einzugliedern, hinzufügt: , die nach dem Vorsatz berufen sind火. Auch der

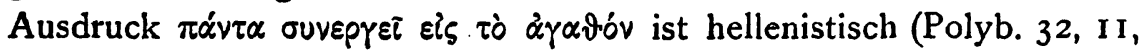
14; Plut. Eroticus 23 p. $769 \mathrm{D})$.

Vor allem ist dem Gedanken von der göttlichen Vorsehung, den wir zuerst heraushören, ein immanent-psychologisches Element beigemischt: wo Liebe zu Gott als dem höchsten Gut ist, da verschieben sich von selbst die Werte und Werturteile so, daß alles, was uns begegnet, in der Richtung des Guten mithelfen muß. So unmittelbar praktisch dieser Gedanke für die Gegenwart ist - er ist nicht der eigentlich paulinische Vorsehungsglaube.

Dort steht vielmehr der alttestamentlich-prophetische Gedanke der Erwählung zum Heil im Mittelpunkte, aus der dann alles weitere mit Notwendigkeit folgt (Röm 8, 29. 30. 31). Insbesondere spielt bei Paulus der syllogismus practicus a majore ad minus eine große Rolle (nicht nur Röm 8, 3r; 5, 6-10, sondern auch sonst, ausgesprochen und unausgesprochen): nach alledem, was wir Christen $\mathrm{s}$ chon erlebt haben an Gnadenbeweisen Gottes, vor allem nach seiner großen Heilstat im Opfer seines Sohnes, ist das, was uns noch fehlt und was wir noch von ihm erwarten.möchten, etwas so Geringes, daß es eine Beleidigung Gottes wäre, ihm nicht auch dies 
noch zuzutrauen. Dies ist diejenige Form des christlichen Vorsehungsglaubens, die A. Ritschl als dèn eigentlichen Kern der christlichen Religion verstehen gelehrt hat: er fußt nicht so sehr auf dem Glauben an den Schöpfergott mit dem optimistischen Motto:

-Was unser Gott geschaffen hat,

das wird er auch erhaltene

sondern auf der Erfahrung von der Erlösung

Ist Gott für uns, wer mag wider uns sein?

Hat er doch seines eignen Sohnes nicht verschont;

Wie sollte er uns mit ihm nicht Alles schenken?

Dies ist das eigentlich religiöse Grundgefühl bei Paulus, dem er in sehr mannigfaltiger Weise Ausdruck gibt; ich hebe nur den sehr

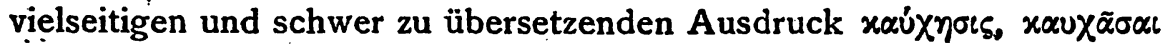
(z. B. Röm 5, 2) hervor, der etwa bedeutet: sreligiöses Hochgefühl, Zuversicht, Frohlocken, ,Trotzen' auf Gott, wie Luther sagt. Oder jene herrliche Stelle 2. Kor 4, $8 \mathrm{f}$.

allezeit bedrängt, aber nicht in die Enge getrieben,

in Verlegenheit, aber nicht in Verzweiflung

verfolgt, abir nicht verlassen,

$\mathrm{Zu}$ Boden geworfen, aber nicht zu Grunde gerichtet.

Diese männlich-heroische Tapferkeit, in der der Apostel großes geleistet und gelitten hat, diese Paradoxie der Weltüberwindung, mag sich auch in die rein religiöse Stimmung leicht ein wenig von der selbstbewußten Haltung des stoischen Weisen einmischen, ist und bleibt doch die ewige Seele der christlichen Religion; und etwas von dieser freudig-trotzigen, entschlossenen und sieghaften Stimmung muß da vorhanden sein, wo noch der Name Christentum berechtigt sein soll. Diese Haltung darf nicht verwechselt werden mit der optimistischen Vertrauensseligkeit der Aufklärungszeit - ihr ist durch das moderne Weltbild für immer der Todesstoß versetzt - dies Vertrauen ruht auf der aus Erfahrung gewonnenen Überzeugung, das in unserem Leben ein nicht von uns ersonnener $\mathrm{Pl}$ a $\mathrm{n}$. ist, der auf unsre geistige und sittliche Vollendung abzielt, daß alles äußere Mißgeschick, alle Hemmung und Demütigung, die wir erfahren, nicht dagegen spricht, daß de $\mathrm{n}$ och und trotzdem dieser gute Wille, der über uns waltet, in irgendeiner Weise zum Ziel kommen muß, wenn wir uns ihm hingeben und auf ihn eingehen. Wer diesen kühnen Glauben wirklich empfinden soll, muß freilich - ebenso wie Paulus - in seinem Leben irgendwelche Erfahrungen davon gemacht

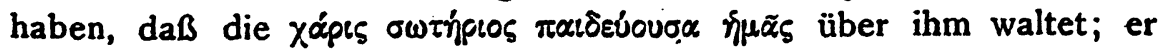
muß die.Spuren göttlicher Lebensführung beobachtet haben. So viel 
Innerlichkeit, so viel Lauschèn auf den Sinn des eignen Lebens freilich muß von dem erwartet werden, der im Sinne des Paulus sein religiöses Leben gestalten soll. Wie denn überhaupt eine so tiefe Religiosität wie die des Apostels wohl niemals von dem mehr oder weniger oberflächlichen Durchschnitt der Menschen nacherlebt werden wird, sondern nur von wenigen. Ich wollte nur zeigen, daß jener syllogismus practicus aus den bereits erlebten Heilserweisungen auf die in der Zukunft noch $\mathrm{zu}$ erlebenden das innere Wesen dieses Typus von Religion ist und überall vorhanden sein muß, wo er weiterleben soll.

d) Dieser Vorsehungsglaube hat nun zum Gegenstand den eigentlich christlichen Gottesbegriff, wie er sich dem Apostel unter dem Eindruck der Sendung und des Liebesopfers Christi gestaltet hat. In ihm ist die $\mathrm{Gn}$ a d e der vorherrschende $\mathrm{Zug}$, und das heißt nicht weichlich-schwächliche Milde eines gutherzigen Menschenvaters, sondern der entschlossene und durch nichts abzulenkende Liebeswille, seine Erwählten zur vollendeten Gemeinschaft mit ihm, zur "Freiheit v von der Vergänglichkeit, von der Knechtschaft des Fleisches und der Sünde, zur »Herrlichkeit der Kinder Gottes « hinanzuführen. Daß Gott die Sünden vergibt, »erläßt «, daß er sgeschenkweises ( $\delta \omega \rho \varepsilon \alpha \dot{v})$ die Gerechtsprechung erteilt, ist uns ein Mittel zu diesem Zweck, die Aufhebung der Trennung durch die Schuld nur ein Schritt auf dem Wege der völligen „Zusammenführung * (d. i. น $\alpha \alpha \lambda \lambda \alpha \gamma \dot{\eta})$, des vollendeten "Friedens\& zwischen Gott und Mensch. Dieser Begriff von Gott als der zielbewußten Willensmacht, die uns $z u$ 'vollendeter Heiligkeit, d. h. Reinheit und Göttlichkeit führen will, ist das unverlierbare Ergebnis des religiösen Erlebens und Denkens des Paulus, und sofern man überhaupt noch von Christentuma soll reden können, muß diese Überzeugung davon in irgendeinem Maße vorhanden sein. Ja, man kann auch sagen: überall, wo der Glaube vorhanden ist, daß dies der Sinn und das Ziel des Weltgeschehens ist, da ist auch noch Christentum im Sinn des Paulus vorhanden, wie bunt verkleidet oder wie blaß abgetönt auch die Gedanken erscheinen mögen.

2. Wir kommen damit auf den zweiten Hauptpunkt: Das Verhältnis des Menschen zu Gott, wie es sich nach paulinischem Denken gestaltet. Der Gedanke, auf den hier alles ankommt, versteckt sich in der antipharisäischen Rechtfertigungslehre, die uns fremd geworden ist, weil der Anlalb zu ihr in unsrer Welt nicht mehr vorhanden ist. Wir können nicht mehr rechnen und brauchen nicht mehr $z \mathfrak{u}$ rechnen mit der jüdischen Vergeltungslehre, wonach 
der Mensch auf Grund seines Tuns an Gott Forder ung en stellen kann und darf (die xxúxyors im pharisäischen Sinne Röm 3, 27). So weit hat der Protestantismus im Zeichen des Paulus gesiegt, daß niemand ernstlich mehr den Satz zu verfechten wagen wird, Gott sei um seiner Gerechtigkeit willen verpflichtet, menschliches Tun mit Heil zu lohnen: $\mathrm{Zu}$ tief ist der Gedanke durchgedrungen, daß der Mensch Gott gegenuiber immer nur der Empfangende sein kann, der völlig von ihm Abhängige, dem allein Gebet und Dank ziemt, aber nicht Pochen auf die eigne Leistung. Auch die neue christliche

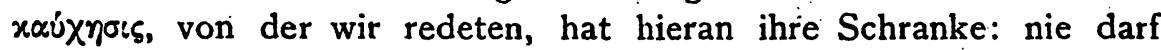
und kann sie sich zu einem Fordern oder zu einem wirklichen Trotz gegen Gott steigern, ohne sofort ihre innere Berechtigung zu verlieren. Daß Gott sein Heil schenkt ( $\delta \omega p \varepsilon \alpha \dot{v}$ ), aus Liebe und Gnade, nicht aus Gerechtigkeit, das ist der Kern der Rechtfertigungslehre. Wenn wir von ihrer scholastisch-juristischen Form absehen, die dem Paulus unvermeidlich war, weil er mit pharisäischen Judaisten zu kämpfen hatte, und mit ihm die Formel: Rechtfertigung aus Werken oder Glaube vorgeschrieben wär, so bleibt übrig der Gedanke Jesu, daß das Reich Gotíes berechnet sei auf Kindesnaturen, daß es bestimmt ist für Arme, Hungernde, Empfängliche, und daß es sich sowohl den Schriftgelehrten wie den Musterfrommen verschließt, die Gott gegenüber auf ihre Leistungen protzen, wie jener Pharisäer im Gleichnis. Damit haben wir eine der tiefsten, innern Übereinstimmungen entdeckt zwischen Paulus und Jesus, die nur durch die Abweichung in der Terminologie verhüllt ist. Es ist ein Verhängnis, daß dieser im Grunde so schlichte Gedanke durch die jüdisch-pharisäische Fragestellung, auf welche Weise der Mensch gerecht gesprochen werde, für den modernen Menschen verdunkelt ist; daß eine $\mathrm{jur}$ is t is che Formel gebraucht wird; um auszudrücken, daß das Verhältnis zwischen Gott und Mensch aus der Sphäre des Rechtlichen ein für allemal herausgehoben sein soll.

Die übelste Folge davon ist, daß der Glaube bei dieser Förmulierung immer wieder als eine $\mathrm{L}$ e istung erscheinen muß, die Gott verlangt und auf Grund deren man nun auch das Heil fordern kann. Während doch Paulus das Gegenteil meint und sagen will. Es ist eine der wichtigsten Aufgaben der modernen Predigt und des Unterrichts, den wahren Sinn des Wortes ,Glaube* der Christenheit klarzumachen: er ist das Gegenteil von Leisten und Tun, ein Sichhingeben, ein Sichschenkenlassen, ein dankbares Sichführenlassen, ein Ergreifen der dargebotenen Hand und das tapfere Eingehen auf seinen Willen. 
Wer es verstünde, unserm Volke den Glauben in diesem Sinne wieder $\mathrm{zu}$ predigen, verständlich und vertraut $\mathrm{zu}$ machen, der würde nicht nur Paulus sondern den innersten Sinn auch der Verkündigung Jesu zu Ehren bringen und zum Leben erwecken.

3. Der Christusglaube und die Christusmystik des $\mathrm{Paulus}$ werden, wenn man sie historisch richtig interpretiert, vielen modernen Christen wohl für immer fremd bleiben. Denn des Apostels Verhältnis zu dem erhöhten Herrn ist ein durchaus supranaturales, auf Gebet und Wunderwirkung berechnetes: a) Praktisch genommen, steht Paulus zu dem ,Herrn* wie zu Gott, von ihm erwartet er "Gnade und Friedes wie von dem himmlischen Vater; zu ihm betet er wie zu dem allmächtigen Gotte, von ihm empfä̀ngt er übernatürliche Wunderwirkungen, Kraft und Mut strömt von ihm aus in sein Herz, im Leiden erfährt er das sieghafte s.Leben Jesu<, im Kampf mit der Sünde die Hilfe des Geistes Christi. Es gibt gewiß viele Christen, die überzeugt sind, daß wir ganz genau so zu dem Herrn Christus beten und in einem lebendigen und fruchtbaren Verkehr mit ihm stehen. Unsre liturgischen Gebete, unsere kultische Sprache geben diesem Glauben immer neue Nahrung; ja sogar die christliche Umdeutung des Alten Testaments, wie Paulus sie geübt hat, lebt unter uns ungebrochen fort. „Der Herr ist mein Hirt - von wie vielen wird dieser Psalm als ein Gebet zu Christus gesprochen und gefühlt. Aber es wäre wiederum eine Aufgabe für eine sehr zart anzufassende religiöse Enquête, einmal festzustellen, inwieweit diese Christus-Frömmigkeit eine klar bewußte ist, und ob nicht dieser naive Modalismus Ist es wirklich in vollem Ernst Christus, an den sich das Gebet richtet, und denkt man nicht in Wahrheit an Gott dabei? Das Ergebnis solcher Nachfrage wird vermutlich sehr mannigfaltig sein. Es gibt zweifellos viele protestantische Christen, die mit voller Überzeugung und alles Ernstes zu Christus beten, wie es viele Katholiken mit dem Gebet zur Jungfrau und zu den Heiligen ganz ernst meinen. Aber ebenso werden andere, wenn man sie fragt, sofort eine gewisse Unklarheit zugeben und fortan schärfer unterscheiden. Und im ganzen wird der moderne Christ geneigt sein, das Gebet zu Christus aufzugeben zugunsten des Gebetes an den himmlischen Vater. Denn die Vorstellung des Erhöhten, auch da wo sie theoretisch zugegeben wird, wird bei einem modern denkenden Christen niemals die unmittelbar überzeugende Kraft wieder gewinnen, die sie für das älteste Christentum, insbesondere für Paulus besaß. Von nationalistischen Bedenken abgesehen: wir stehen zu sehr unter dem Einfluß des Jesus- 
Bildes der Evangelien - jedenfalls weit stärker als Paulus -; damit aber tritt die menschlich-geschichtliche Gestalt Jesu, wie göttlich und verklärt sie auch gedacht werden möge, in den Vordergrund; und an sie wird sich immer viel mehr Ehrfurcht und Vertrauen, ein ethisches Abhängigkeitsverhältnis anschließen, als ein rein religiöses Gebetsverhältnis. Gewiß kann auch jenes leicht die Form des Gebetes annehmen, aber dies wird immer ein uneigentliches bleiben und sich von dem wirklichen Gebet zu Gott unterscheiden. Dies alles gilt, wie gesagt, von dem smodernen * Christen; ich leugne nicht, daß es viele Christen auch heute gibt, die ohne alle Bedenken zu dem erhöhten Christus beten. Im großen und ganzen aber hat die Gestalt des - Herrne für die meisten Christen heute nur noch die Bedeutung, daß. sie mahnend und gewissenweckend, tröstend und anfeuernd als ein ehrfürchtig verehrter Gefährte unseres Lebens mit uns geht, eine lebendige persönliche Vergegenwärtigung des heiligen und gnädigen Gotteswillens. Auch dies mag man ein religiöses Verhältnis nennen; gegenüber dem Christus-Glauben des Paulus aber bedeutet es eine Abschwächung; aụs dem Kultus des erhöhten Christus ist ein ethischreligiöses Verhältnis zu Yesus geworden. Das Bekenntnis > Jesus lebt « wird von vielen Christen nicht mehr in dem eigentlichen transzendenten und wunderhaften Sinne empfunden, sondern in dem diesseitigen, daß die Gestalt Jesu und seine Art des Lebens mit Gott und für Gott als eine moralische Macht über unser Leben empfunden wird, der wir uns nicht entziehen können. Der \Glaube an Jesus « nimmt überwiegend die Gestalt der >Nachfolge Jesu * an. Freilich fehlt es auch bei Paulus nicht an dieser Form: viel stärker als es gewöhnlich

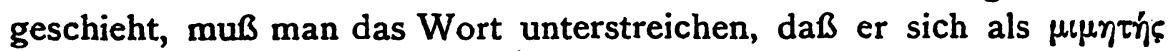
Christi fühlt ( 2 Kor $I_{1}, \mathrm{I}$ ). Und weit mehr, als die heutige Theologie zugeben will, verbindet sich auch bei ihm mit der Vorstellung des Erhöhten die Anschauung des Menschen Jesus, der svom Weibe geboren und unter das Gesetz getane im sGehorsam bis zum Todec und in der Liebe zu andern sich selbst geopfert hat. Aber richtig ist, daß die Gedanken nicht entfernt die Bedeutung für ihn haben, wie die eigentlich religiöse Christus-Frömmigkeit, der Kultus des Erhöhten ${ }^{1}$.

b) Dies zeigt sich besonders an dem Überwiegen der ChristusMystik. So zahllos oft die Formel sin Christo im Kultus und der Einzelfrömmigkeit nachgesprochen wird, so selten ist sie doch im vollen historischen Sinne nachempfunden. Denn das in Christus seine bedeutet doch bei ihm ein völliges Verschmelzen mit dem

\footnotetext{
1 Vgl. meine Schrift: ,Jesus im Glauben des Urchristentamse. Tübingen 1910.
} 
himmlischen Herrn zu einer mystischen Einheit, wobei dann freilich die scharfen Umrisse der personalistischen Vorstellung sich erweichen und der Gedanke des alldurchdringenden Christus-Geistes (2 Kor 3, 17) sich einstellt. Für Paulus war die metaphysische Gleichsetzung der Person Christi mit dem unpersönlichen Pneuma möglich, weil die antike Denkweise, in der er aufgewachsen ist, überhaupt sehr leicht die Grenze zwischen abstraktem Begriff und Persönlichkeit überschreitet ${ }^{1}$, und weil das Bild der evangelischen Überlieferung auf ihn noch nicht die Macht ausübte, wie auf uns. Aber aus diesem Grunde wird wahre Christus-Mystik, d. h. diejenige Form religiösen Empfindens, bei der die Persönlichkeiten des Christen und Christi inéinanderfließen, eine Seltenheit sein. Man darf sich nicht dadurch täuschen lassen, daß jene mystische Formel wie alle mystischen Klänge auf viele einen berauschenden, ich möchte fast sagen: musikalischen Zauber ausüben, so daß sie in erhöhter religiöser Stimmung gerne danach greifen: es fragt sich aber, ob dem ein wirkliches Empfinden entspricht. Auch hier würde eine religiöse Enquête, wenn sie möglich wäre, vielleicht ein negatives Ergebnis haben.

Aber man muß sogar zweifeln, ob Paulus selber die mystische Formel, die ihm aus seiner hellenistischen Umgebung zugeflossen ist ${ }^{2}$, so ernst gemeint und so voll empfunden hat, wie unsre Exegese es behauptet. Es ist doch sehr bemerkenswert, daß er an der Stelle, wo er sie am schärfsten und prinzipiellsten ausspricht (Gal 2, 20):

>Mein Ich lebt nicht mehr: Christus lebt in mir * sofort die Interpretation hinzufügt:

- Was ich aber noch lebe im Fleisch, das lebe ich im Glauben an den Sohn Gottes, der mich geliebt und sich für mich dahin gegeben hat. *

Er ersetzt hier die mystische Formel durch eine Beschreibung, in der nicht nur die volle Persönlichkeits-Vorstellung von Christus wieder auflebt, sondern auch die subjektive religiöse Funktion ganz anders erscheint: an Stelle des Aufgehens des Ich in Christus, an Stelle der völligen passiven Aufsaugung durch ihn, an Stelle der mystischen Formel tritt die Sprache der Ich- und Du-Religion, das dankbare Anschauen der Liebestat Christi, das Ergreifen seiner Gnade, das SichAufrichten an seinem heiligen und gütigen Willen. Diese Interpretation erscheint mir sehr bedeutsam und erweckt in mir den $\mathrm{Z}_{\text {weifel, }}$

1 Vgl: meine Schrift, Christuse Tübingen 1909. i920.]

2 Vgl. Reitzenstein, Die hellenistischen Mysterienreligionen. Leipzig 1910. [2. Aufl. 
ob jene mystische Formel das Innenleben des Apostels wirklich zutreffend beschreibt. Liegt hier nicht einer jener zahllosen Fälle von Anpassung an überlieferten religiösen Ausdruck vor, wie sie zu allen Zeiten in der Kirche unvermeidlich sind? Jeder, der eine überkommene Form religiösen Ausdruckes auf sich anwendet, muß sie dem eigenen Empfinden adaptieren; hat nicht auch Paulus die hellenistische Formel hier in die Form israelitisch-christlicher Frömmigkeit zurückverwandelt?

Jedenfalls enthält Gal 2, 20 eine Anleitung, wie auch die ChristusMystik im modernen Christentum lebendig gemacht werden kann, freilich unter Verzicht auf die eigentlich mystische Form. Diesen Weg hat schon "Johannese beschritten, denn bei ihm bedeutet sLeben in Christus « nicht mehr eigentliche mystische Verschmelzung, sondern das Beziehen der gesammten Religion und des sittlichen Lebens auf Christus. In seinem ideal verklärten Bilde die \Gnade und Wahrheit* Gottes anschauen, an ihm zur Gotteserkenntnis gelangen, ihn als den Führer immer vor Augen haben, in sseiner Liebe bleiben «, seine „Gebote " tun und wie er dadurch in der Liebe Gottes bleiben (Joh I5, Io). Diese Konzentrierung des inneren Lebens auf die Gestalt Jesu, mit ihm leben, in seinem Sinne wirken, sein Wesen widerstrahlen im eignen Leben - das ist eine Form der Christus-Mystik, die auch dem modernen Menschen möglich ist. Insbesondre ist die Aufgabe des »mit Christo Leidens * eine Parole, die niemals in der Christenheit verstummen kann. Mag sie auch des mystischen Untergrundes, den sie bei Paulus hat, beraubt sein, sie wird immer die höchste Probe des Christentums bleiben. Und so dürfen wir sagen: wenn auch das moderne Christentum mehr in der Form der Gotteskindschaft und des Glaubens an Gott sein Gepräge haben wird - wie Jesus sie gelebt und gelehrt hat - so wird es doch immer dabei bleiben können, daß wir uns als Jünger Jesu oder als -Knechte Christis fühlen - dies letztere weniger im Sinne der metaphysischen Abhängigkeit von dem weltherrschenden erhöhten Herrn, sondern in dem ethischen Sinne, daß wir nicht mehr uns selbst sondern für ihn und sein Werk leben.

4. Das führt uns zu einem letzten kurzen Wort über die Ethik des Paulus. Mag uns der Zugang zu ihr durch die asketisch-dualistischen Elemente erschwert werden, mögen mit dem Wegfall der eigentlich eschatologischen Stimmung viele seiner Forderungen nur in andrer Tonart von uns aufgenommen werden können - es bleibt genug Positives, genug männliche Kraft, Selbstzucht und Heroismus in der paulinischen Ethik, um auch moderne Christen zur Aufgabe 
der Weltverklärung und Durchsetzung des Willens Gottes in der Welt begeistern zu können.' Es ist besonders wieder der stoische Einschlag in dieser Ethik, seine Betonung der Freiheit (1 Kor 6, 12) und der königlichen Weltbeherrschung (I Kor 3, 3 I ff.), die griechischen Akzente gewisser Forderungen, z. B. der $\tau \iota \mu$ ' (I Thes 4, 4) und

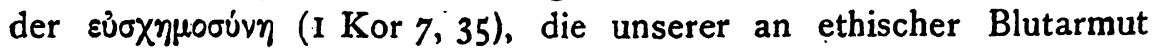
leidenden Zeit Eisen zuführen können. Vor allem ist noch lange nicht genügend verstanden und ausgeschöpft, was Paulus von der $\mathrm{Li}$ e b e unserem Geschlecht zu sagen hat, geschweige denn daß von der Verwirklichung seiner Forderungen geredet werden könnte. Die ungeheure Fülle vielseitiger praktischer, nüchterner, weiser Mahnungen;

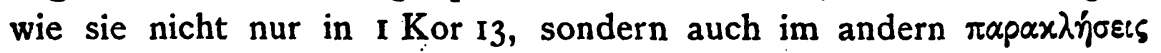
(Röm 12; Kol 3) ausgebreitet vor uns liegt, ist noch nicht annähernd in Praxis umgesetzt, ja noch nicht einmal in eine packende theoretische Unterweisung. Wenn er von der Liebe weiter nichts gesagt hätte, als das Wort:

sie freuet sich nicht an der Ungerechtigkeit

sie freuet sich an der Wahrheit

so hätten wir schon daran ein ethisches Regulativ, das an Erhabenheit und Fruchtbarkeit gar nicht zu überbieten ist. Freilich steht dies alles bei ihm unter den Zeichen: sich vermag nichts ohne den, der mich stark macht, Christus «, und alles, was der Christ Gutes haben und tun kann

\author{
Liebe, Freude, Friede, \\ Geduld, Freundlichkeit, Gütigkeit, \\ Glaube, Sanftmut, Keuschheit
}

gilt ihm in letzter Linie als eine ,Frucht des Geistes * (Gal 5, 22), als eine wunderbare Gnadenwirkung Gottes; es ist und bleibt eine durch und durch religiöse Ethik. Aber dabei muß es im Christentum bleiben. Mag der moderne Christ sich das weniger transzendent, weniger wunderhaft denken: so lange er noch Wert darauf legt, Christ zu sein, wird auch er sich bewußt bleiben, daß alles Tun des Guten für ihn nur möglich ist, wenn er sich in beständiger innerer Fühlung mit Gott und Christus hält, seine Liebe zu den Brüdern wird immer Ausstrahlung sein der innig empfundenen Liebe Gottes. 